\title{
The angular momentum of twisted light in anisotropic media: chiroptical interactions in chiral and achiral materials
}

Kayn A. Forbes, David L. Andrews

Kayn A. Forbes, David L. Andrews, "The angular momentum of twisted light in anisotropic media: chiroptical interactions in chiral and achiral materials," Proc. SPIE 10672, Nanophotonics VII, 1067210 (4 May 2018); doi: $10.1117 / 12.2305723$

SPIE. Event: SPIE Photonics Europe, 2018, Strasbourg, France 


\title{
The angular momentum of twisted light in anisotropic media: chiroptical interactions in chiral and achiral materials
}

\author{
Kayn A. Forbes and David L. Andrews* \\ School of Chemistry, University of East Anglia, Norwich NR4 7TJ, United Kingdom
}

\begin{abstract}
The issue of whether the optical orbital angular momentum of light can play any significant role in chiroptical interactions has seen a resurgence of interest in the past few years. Revising preliminary expectations, it has been shown both theoretically and experimentally that the topological charge can indeed play a decisive role in some chiroptical interactions, with the rates of these optical phenomena proving sensitive to the sign of the vortex charge $\ell$. Using quantum electrodynamics, it is now revealed how the inclusion of molecular electric-quadrupole transition moments in both chiral and achiral anisotropic media produces such an effect. Specifically, for single-photon absorption it transpires that both the orbital and spin angular momentum must be engaged through a circularly polarized vortex beam. The chiroptical effect is identified as a manifestation spin-orbit interaction in light.
\end{abstract}

Keywords: Chirality, twisted light, optical vortex, nanoparticle, photon interactions, optical activity, orbital angular momentum, spin-orbit coupling, chiroptical effect

\section{INTRODUCTION}

It is now well established that suitable tailored laser light can possess both an orbital and a spin angular momentum (OAM and SAM, respectively). ${ }^{1}$ The SAM of light, with helicity eigenvalues restricted to $\pm \hbar$ per photon manifest in circularly polarized states, ${ }^{2}$ is well-known and utilized in numerous areas of optics. ${ }^{3,2}$ In contrast to the SAM of light, the OAM associated with the different forms of structured light has no such bounds on dimensionality, each photon being able to possess values of $\pm \ell \hbar$, where $\ell$ is any integer. ${ }^{4}$ The quantity $\ell$ represents the topological (or vortex) charge that characterizes the wavefront structure: the higher the value, the more 'twists' the beam exhibits within a wavelength. The burgeoning field of optical orbital angular momentum has shown an unrelenting growth since its inception, both fundamentally progressing in an upward sense, and equally broadening across a plethora of sub-disciplines throughout optics and beyond. ${ }^{5,6}$ Researchers have vacillated for over a decade on the question of whether the orbital angular momentum of light can play any decisive role in chiroptical interactions, and the results from a spate of recent theoretical and experimental studies indicate a need to revise some of the conclusions drawn from rudimentary work in the field.

Chiroptical interactions involve the interplay of molecular and radiation components within a total light-matter system to produce optical rates and forces that are sensitive to changes in handedness. ${ }^{711}$ For the material part of the total radiationmatter system, the molecules are said to be chiral if they have no improper axis of rotation, i.e. they can exist in the form of either one of two mirror images that are non-superimposable, and so are designated either right- or left-handed. The chirality of light can be manifest in two ways: the sense of direction that the electromagnetic field vectors are rotating in a circularly polarized beam, being either clockwise (right-handed) or anti-clockwise (left-handed); or the sense of direction

Nanophotonics VII, edited by David L. Andrews, Angus J. Bain, Jean-Michel Nunzi, Andreas Ostendorf, Proc. of SPIE Vol. 10672, 1067210 - @ 2018 SPIE · CCC code: 0277-786X/18/\$18 · doi: 10.1117/12.2305723 
that the helical wavefront is twisting in a beam possessing orbital angular momentum: beams possessing $\ell>0$ are lefthanded, whilst $\ell<0$ are right-handed.

Earlier theoretical studies on chiroptical light-matter interactions with structured light were restricted to the dipole approximation, neglecting quadrupole transitions and other higher-order couplings between material and radiation. ${ }^{12,13}$ Likewise, the limited experimental efforts ${ }^{14,15}$ were restrictive on certain aspects which now appear to be essential if one wishes to observe a sensitivity to the vortex twist (for example through the interactions of circularly polarized vortex beams with anisotropic media). It is important to realize, however, that these initial experimental studies verified subtleties of principle determined in both the earlier and latest theoretical formulations. Contemporary efforts have centered on studying single-photon absorption in achiral media, where it has been shown that by using a circularly polarized vortex beam, the total angular momentum of light does indeed play a chiroptical role. ${ }^{16,17}$ In contrast, however, another recent experimental study observed that by exploiting plasmonic enhancement ${ }^{18,19}$, one can expect a chiroptical effect in chiral media solely due to the orbital angular momentum of the beam. ${ }^{20}$ The latter phenomenon, which uses linearly polarized light, has been termed 'helical dichroism'. A further recent study, using a quantum electrodynamics (QED) methodology, has determined that by inclusion of electric quadrupole transitions, the rate of single-photon absorption in oriented chiral media is sensitive to the circular polarization state, the handedness of the topological charge, and that of the molecules themselves - a process that has been termed circular vortex dichroism (CVD) ${ }^{21,22}$, due to its similarity to circular dichroism (CD).

In this paper it will be highlighted how the same QED analysis that secured the CVD result in refs ${ }^{21,22}$ also elicits a discriminatory single-photon absorption solely attributable to an interplay of handedness between the circular polarization and the vortex twist i.e. a CVD or CD-like effect in achiral molecules. It will be shown how this effect originates from an electric quadrupole-electric quadrupole (E2E2) mechanism, in contrast to the electric dipole-electric quadrupole (E1E2) interference mechanism of the previously reported CVD effect.

\section{THEORY}

To study the interaction of a twisted beam of light interacting with discrete optical centers we can employ the PowerZienau -Woolley (PZW) interaction Hamiltonian ${ }^{23,24}$ cast in its multipolar form as

$$
H_{\mathrm{int}}^{\mathrm{mult}}=\sum_{\xi}\left[-\varepsilon_{0}^{-1} \boldsymbol{\mu}(\xi) \cdot \boldsymbol{d}^{\perp}\left(\boldsymbol{R}_{\xi}\right)-\varepsilon_{0}^{-1} Q_{i j}(\xi) \nabla_{j} d_{i}^{\perp}\left(\boldsymbol{R}_{\xi}\right)-\ldots-\boldsymbol{m}(\xi) \cdot \boldsymbol{b}\left(\boldsymbol{R}_{\xi}\right)-\ldots\right]
$$

where the term involving $\boldsymbol{\mu}(\xi)$ is the E1 electric dipole coupling for a molecule $\xi$; the second term involving the electric quadrupole moment $Q_{i j}(\xi)$ is the E2 coupling, and the final term is the magnetic dipole $\boldsymbol{m}(\xi)(\mathrm{M} 1)$ interaction. Retaining the E1 term alone is tantamount to invoking the well-known electric-dipole approximation, prevalent throughout molecular optics. It should be noted that to this level of multipole moment expansion there is an additional diamagnetic interaction term. However, the term is quadratic in the magnetic field and, thus, only contributes to optical processes where the number of photons involved is two or more; as such, its inclusion is unnecessary in the theory of single-photon absorption. ${ }^{25}$

To take account of the properties of structured light, the well-known vacuum mode expansions for the transverse electric displacement field $\boldsymbol{d}^{\perp}$ and the magnetic field $\boldsymbol{b}$ need to be recast from their more familiar plane-wave form. We confine our analysis to the paraxial approximation, whereby it is legitimate to assume that the laser light propagation consists of wave vectors that have negligible inclination to the optical axis. As such, one of the most widely utilized solutions to the paraxial wave equation are the Laguerre-Gaussian modes - accordingly, these are the most common modes for generating and describing laser beams with an orbital angular momentum. The electric displacement and magnetic field expansions 
for LG beams in the paraxial approximation emerge as the following functions of the cylindrical coordinates: ${ }^{26}$ the off-axis radial distance $r$, axial position $z$, and azimuthal angle $\phi$;

$$
\begin{aligned}
& \boldsymbol{d}^{\perp}(\boldsymbol{r})=i \sum_{\boldsymbol{k}, \eta, \ell, p}\left(\frac{\hbar c k \varepsilon_{0}}{2 V}\right)^{1 / 2}\left[\boldsymbol{e}_{\ell, p}{ }^{(\eta)}(\boldsymbol{k}) a_{\ell, p}{ }^{(\eta)}(\boldsymbol{k}) f_{\ell, p}(r) \mathrm{e}^{(i k k+i \ell \phi)}-\overline{\boldsymbol{e}}_{\ell, p}{ }^{(\eta)}(\boldsymbol{k}) a_{\ell, p}^{\dagger(\eta)}(\boldsymbol{k}) \bar{f}_{\ell, p}(r) \mathrm{e}^{-(i k z+i \ell \phi)}\right], \\
& \boldsymbol{b}(\boldsymbol{r})=i \sum_{\boldsymbol{k}, \eta, \ell, p}\left(\frac{\hbar k}{2 \varepsilon_{0} c V}\right)^{1 / 2}\left[\boldsymbol{b}_{\ell, p}{ }^{(\eta)}(\boldsymbol{k}) a_{\ell, p}{ }^{(\eta)}(\boldsymbol{k}) f_{\ell, p}(r) \mathrm{e}^{(i k z+i \ell \phi)}-\overline{\boldsymbol{b}}_{\ell, p}{ }^{(\eta)}(\boldsymbol{k}) a_{\ell, p}^{\dagger(\eta)}(\boldsymbol{k}) \bar{f}_{\ell, p}(r) \mathrm{e}^{-(i k z+i \ell \phi)}\right],
\end{aligned}
$$

where $f_{\ell, p}(r)$ is the radial distribution function for a paraxial beam of waist $w_{0}$

$$
f_{\ell, p}(r)=\frac{C_{p}^{|\ell|}}{w_{0}}\left[\frac{\sqrt{2} r}{w_{0}}\right]^{|\ell|} \mathrm{e}^{\left(-r^{2} / w_{0}^{2}\right)} L_{p}^{|\ell|}\left(\frac{2 r^{2}}{w_{0}^{2}}\right) .
$$

Here, $a_{\ell, p}{ }^{(\eta)}(\boldsymbol{k})$ and $a_{\ell, p}{ }^{\dagger(\eta)}(\boldsymbol{k})$ are the annihilation and creation operators for a photon of mode $(\boldsymbol{k}, \eta, \ell, p)$.

\section{TWISTED BEAMS INTERACTING WITH MATTER}

In order to highlight the mechanism in which the handedness of a vortex beam plays a role in chiroptical interactions with both chiral and achiral media, we shall concentrate on basic optical process of single-photon absorption. Consider a ground state molecule absorbing a photon from a single mode of an LG beam, where the initial and final states of the moleculeradiation system are $|i\rangle=\left|E_{0}\right\rangle|n(\boldsymbol{k}, \eta, \ell, p)\rangle$ and $|f\rangle=\left|E_{f}\right\rangle|n(k, \eta, \ell, p)\rangle$, respectively. Single-photon absorption is of first-order in the interaction Hamiltonian, and using standard perturbation methods the matrix element can be written as

$$
\begin{aligned}
M_{f i} & =\left\langle f\left|H_{\mathrm{int}}\right| i\right\rangle \\
& =-\varepsilon_{0}^{-1} \mu_{i}^{f 0}(\xi)\left\langle(n-1)\left|d_{i}^{\perp}(\boldsymbol{r})\right| n\right\rangle-m_{i}^{f 0}(\xi)\left\langle(n-1)\left|b_{i}(\boldsymbol{r})\right| n\right\rangle-\varepsilon_{0}^{-1} Q_{i j}^{f 0}(\xi)\left\langle(n-1)\left|\nabla_{j} d_{i}^{\perp}(\boldsymbol{r})\right| n\right\rangle,
\end{aligned}
$$

where the mode-dependence for the radiation state has been dropped for clarity. As has been previously shown, ${ }^{21,22}$ it is the inclusion of the electric quadrupole E2 coupling to the radiation field which produces couplings showing a sensitivity to the sign of $\ell$. This necessity of quadrupole couplings is due to the fact that, to this level of multipole expansion, the E2 moments possesses the unique characteristic being dependent on the gradient of the electric field.

Carrying out all of the E1, M1, and E2 field operations in (5) gives the total quantum amplitude as: ${ }^{27}$ 


$$
M_{f i}=-i\left(\frac{n \hbar c k}{2 \varepsilon_{o} V}\right)^{1 / 2} f_{\ell, p}(r)\left[e_{i} \mu_{i}^{f 0}+\frac{1}{c} b_{i} m_{i}^{f 0}+e_{i} Q_{i j}^{f 0}\left(\hat{r}_{j} \frac{1}{f_{\ell, p}(r)} \frac{\partial f_{\ell, p}(r)}{\partial r}+\frac{1}{r}\left(i \ell \hat{\phi}_{j}-\hat{r}_{j}\right)+i k \hat{z}_{j}\right)\right] \mathrm{e}^{(i k z+i \ell \hat{\phi})} .
$$

The detailed calculations of the gradient operation present in the final term on the right-hand side of (5) can be found elsewhere. ${ }^{22}$ As exhibited in equation (6), there is a clear dependence on the magnitude and the sign of $\ell$ in the phase factor common to all three terms in the square bracket; in addition there is also a linear dependence on $\ell$ in the third term in square brackets: the quadrupole $\mathrm{E} 2$ term.

To calculate the rate of single-photon absorption we are required to substitute the quantum amplitude (6) into the Fermi rule: $\Gamma=(2 \pi / \hbar) \rho_{f}\left|M_{f i}\right|^{2}$, where $\rho_{f}$ is the density of final states. Clearly, this calculation requires the modulus square of the matrix element (6). As such, the dependence on $\ell$ present within the phase factor obviously vanishes: $\left|\mathrm{e}^{i \ell \phi}\right|^{2}=1$. If, as in previous studies ${ }^{12}$, only the E1 and M1 terms of (6) are included, it can be seen that for the rate of single-photon absorption there would be no dependence on the handedness of the vortex beam through the sign of $\ell$. However, due to the inclusion of E2 moments, terms with a dependence on the sign of $\ell$ will survive the squaring of the quantum amplitude. In fact, taking the modulus square of (6), produces a plethora of coupling terms: namely, E1E1, M1M1, E1M1, E1E2, $\mathrm{M} 1 \mathrm{E} 2$, and E2E2 are all interference contributions to the rate when evaluated from the following:

$$
\Gamma=\frac{2 \pi}{\hbar} \rho_{f}\left(\frac{n \hbar c k}{2 \varepsilon_{o} V}\right) f_{\ell, p}{ }^{2}(r)|e_{i} \mu_{i}^{f 0}+\underbrace{\frac{1}{c} b_{i} m_{i}^{f 0}}_{\mathrm{E} 1}+\underbrace{e_{i} Q_{i j}^{f 0}\left(j \frac{1}{f_{\ell, p}(r)} \frac{\partial f_{\ell, p}(r)}{\partial r} \hat{r}+\frac{1}{r}\left(i \ell \hat{\phi}_{j}-\hat{r}_{j}\right)+i k \hat{z}_{j}\right)}_{\mathrm{E} 2}|^{2} .
$$

The E1E1 and M1M1 rate terms represent non-discriminatory (handedness-independent) contributions to single-photon absorption in the dipole approximation ${ }^{24}$, where the M1M1 rate is approximately $10^{-6}$ times smaller than the E1E1 rate. Neither of these terms shows any sensitivity to the sign of $\ell$, nor does either possess the capability to exhibit chiral effects with regards to the circular polarization state or molecular chirality. The E1M1 term is discriminatory, but with regards to the molecular chirality and the SAM manifest through circularly polarized photons: it is the term that gives rise to standard circular dichroism (CD). ${ }^{28}$ All the remaining terms involve at least one E2 interaction; in fact, for any chiroptical phenomenon to exhibit sensitivity to the sign of $\ell$ requires, at minimum, the engagement of electric quadrupole moments.

At this stage it is necessary to consider the symmetry conditions that permit or preclude the registration of chirality by any given electric or magnetic multipole. ${ }^{29}$ First, a distinction between permanent (static) and transition moments has to be borne in mind. Permanent moments of either kind may be non-zero provided their symmetry elements transform under the totally symmetric representation of the molecular point group. Essentially, none of the allowed spatial symmetry operations of the molecule may affect the moment under consideration: for example a molecule lacking any symmetry and therefore necessarily chiral - will allow non-zero values for every electric multipole. But for absorption, or any other process involving an electronic transition, the rule is that the product of the initial and final state electronic symmetries (specifically their irreducible representations known as irreps) must contain the symmetry irrep of one or more components of the multipole under scrutiny. An obvious corollary that immediately arises for any centrosymmetic molecule is that since every irrep is of either even or odd parity, there is no transition that can be simultaneously allowed by both E1 (odd parity) and E2 (even parity) moments; the same principle of exclusion applies to E1 and M1 simultaneity. Therefore when 
the modulus square is taken of the matrix element in order to secure an expression for the rate of absorption, E1M2 and E1E2 (' $\mu m$ ' and ' $\mu Q$ ') cross-terms cannot arise. However, for non-centrosymmetric molecules (and indeed for chiral species in general) such terms will indeed be present in the rate equation. Then, since the ' $\mu m$ ' and ' $\mu Q$ ' products have odd parity, they can be supported only by odd-parity combinations of the field vectors. The latter condition usually predicates the use of circularly polarized light; when structured light is involved, the parity of the wave-front has also to be considered.

\subsection{Chiral media}

From the very general result represented by equation (7), it is readily possible to identify features of the interaction that are distinctive and limited to chiral media. As indicated above, we now isolate the relevant E1E2 term corresponding to chiral media, and which also depends on the sign of $\ell$, giving a contribution to the rate of single-photon absorption expressible as

$$
\Gamma_{\mathrm{EIE} 2}^{\prime}(\ell)=\frac{I(\omega) N}{2 c \hbar^{2} \varepsilon_{o}} \frac{1}{r} f_{\ell, p}^{2}(r) e_{i} \bar{e}_{k} i \ell \hat{\phi}_{j}\left(\mu_{k}^{f 0} Q_{i j}^{f 0}-\mu_{i}^{f 0} Q_{k j}^{f 0}\right)
$$

where $I(\omega)$ is the input beam irradiance per unit frequency interval and $N$ is the number of absorbing chiral molecules. Both $\boldsymbol{\mu}^{f 0}$ and $\boldsymbol{Q}^{f 0}$ are real quantities, and therefore the polarization vectors in (8) must be complex quantities in order to secure a non-zero real result. This reveals the significant conclusion that, to the leading order E1E2 interaction, we may only expect to observe a chiroptical response to the handedness of the vortex beam if that same beam is circularly polarized. Strikingly, the OAM of light alone cannot produce a chiroptical effect in single-photon absorption, and only in a synergy with SAM is it possible to engage the vortex handedness in discriminatory molecules with chiral molecules. This optical process is therefore an analogue of circular dichroism (CD), but with beams carrying a topological charge, and has been termed circular-vortex dichroism (CVD) ${ }^{21}$

With the aid of the following identity ${ }^{24}$

$$
e_{i}^{\mathrm{L} / \mathrm{R}} \bar{e}_{k}^{\mathrm{L} / \mathrm{R}}=\frac{1}{2}\left[\left(\delta_{i k}-\hat{k}_{i} \hat{k}_{k}\right) \mp i \varepsilon_{i k m} \hat{k}_{m}\right],
$$

we may now write (8) in a way that allows the rate to be secured in a form where the influence of circular polarization handedness is readily identifiable:

$$
\Gamma_{\mathrm{ElE} 2}^{(\mathrm{L} / \mathrm{R})}(\ell)= \pm \frac{I(\omega) N}{4 c \hbar^{2} \varepsilon_{o}} \frac{1}{r} f_{\ell, p}{ }^{2}(r) \varepsilon_{i k m} \hat{k}_{m} \ell \hat{\phi}_{j}\left(\mu_{k}^{f 0} Q_{i j}^{f 0}-\mu_{i}^{f 0} Q_{k j}^{f 0}\right)
$$

where the \pm designates either left-handed or right-handed polarization, respectively. 
Due to the fact that the CVD process requires circular polarization, any experimental set-up aiming to observe it will correspondingly produce a standard CD response. Therefore, it is more suitable to secure a rate which takes into account both optical features. Casting such a rate in terms of the absolute difference in absorption rates between left- and righthanded circularly polarized photons yields;

$$
\Gamma^{(\mathrm{L})}-\Gamma^{(\mathrm{R})}=\Delta \Gamma_{\mathrm{CD}}+\frac{I(\omega) N}{2 c \hbar^{2} \varepsilon_{o}} \frac{1}{r} f_{\ell, p}{ }^{2}(r) \varepsilon_{i k m} \hat{k}_{m} \ell \hat{\phi}_{j}\left(\mu_{i}^{f 0} Q_{k j}^{f 0}+\mu_{k}^{f 0} Q_{i j}^{f 0}\right)
$$

where $\Delta \Gamma_{\mathrm{CD}}$ includes both the E1E2 and E1M1 contributions to standard CD:

$$
\Delta \Gamma_{\mathrm{CD}}=\frac{2 \pi N}{\hbar}\left(\frac{n \hbar c k}{2 \varepsilon_{0} V}\right) \rho_{f} f_{\ell, p}{ }^{2}(r)\left[\frac{1}{c}\left(\delta_{i k}-\hat{k}_{i} \hat{k}_{k}\right)\left(\mu_{i}^{f 0} \bar{m}_{k}^{\prime f 0}-\bar{\mu}_{k}^{f 0} m_{i}^{\prime f 0}\right)-\varepsilon_{i k m} \hat{k}_{m} k \hat{z}_{j}\left(\mu_{i}^{f 0} Q_{k j}^{f 0}-\mu_{k}^{f 0} Q_{i j}^{f 0}\right)\right],
$$

casting the entire expression in terms of real quantities by adopting a shorthand terminology for the magnetic transition moments: $m_{i}^{\prime f 0}=i m_{i}^{f 0}$.

Key elements of the physics that can be extracted from (11) and (12) includes the validation that when the beam possesses no OAM, any terms dependent on $\ell$ clearly vanish, leaving the standard CD result. Since both the E2 and M1 interactions with the field are of a similar magnitude, the rate of CVD and CD will be alike. However, (11) also highlights the unique linear dependence of CVD on $\ell$. This increasing significance of the E2 interactions as the degree of vortex twist increases can be expected due to the fact that quadrupole transition moments engage with the gradient of the electric field - such an enhanced role of E2 moments has been recognized previously in studies involving atoms. ${ }^{30,31}$

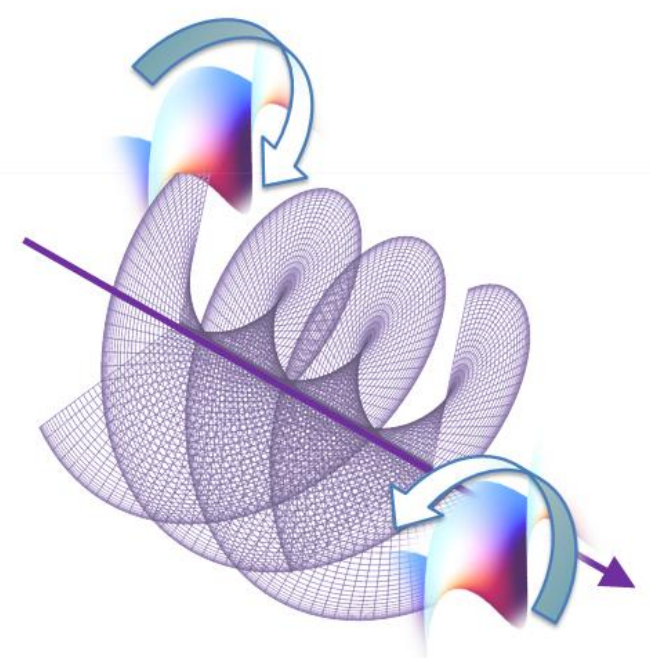

Figure 1: Twisted beam, incident from the left, propagating through an anisotropic system of molecules such as a liquid crystal exemplified by two helical structures with a common orientation. Positioned at opposite sides of the beam, each experiences with regard to its own structure a different directional sense of the phase gradient. 
As it stands, the result (11) is applicable to one or molecules with a fixed orientation at any point within the LG beam. The $\hat{\phi}_{j}$ term contracts with an index of the quadrupole transition moment (which itself has a fixed orientation within the molecule), and therefore the magnitude and sign of the CVD differential will in general vary about the axis of the beam. More specifically, the CVD will vary between a maximum and minimum of opposite sign across the beam profile. The CVD effect therefore registers the varying directions of phase gradient around each intensity ring for an LG beam, as experienced by chiral molecules possessing a common orientation - see Figure 1.

For a system of molecules possessing a locally consistent degree of orientational order, the absorption differential will be enhanced on one side of the beam and equally diminished at exactly the opposite side, as compared to the standard rate of $\mathrm{CD}$ along the singular core. However, due to the radial and azimuthal components being symmetrical about the $z$ axis, any observation over the whole beam profile will leave only terms that are solely dependent on $\hat{z}$ (out of those dependent linearly on the unit vectors): i.e. the standard CD terms. Therefore, when observing the whole beam profile, the CVD has a vanishing net result: it is to be recognized that the effect itself may be deemed local, being exhibited only when the extent of absorption is resolved at different locations within the beam profile.

Finally, it is worth noting that extension of the theory to allow for isotropic media shows that any CVD-effect vanishes upon a rotational average of (11). ${ }^{21}$ Indeed, the only term of (11) that survives an average is the E1M1 contribution explicitly shown in (12) - which is in full agreement with the well-known fact that it is the electric-magnetic interference term that contributes to standard CD in isotropic media. ${ }^{28,24}$

\subsection{Achiral media}

The process of CVD discussed above relies on an interplay between material and radiation chirality to produce a chiroptical effect. This is in the traditional paradigm of discriminatory interactions in light-matter interactions, where the handedness of both the molecules and the light introduce chiral sensitivity to optical phenomena. However, a more contemporary field of research exists wherein chiroptical effects can be observed when light interacts with material that is achiral. ${ }^{32}$ Such interactions are not new, however, with classic examples of chiroptical interactions in achiral media being magnetic $\mathrm{CD},{ }^{33}$ induced CD, ${ }^{34}$ and optical rotation. ${ }^{35}$ Recent experiments of specific interest to us here include the observation of CD using twisted light in non-chiral atomic matter and nanostructures. ${ }^{17,16}$ Needless to say, chiral species may also exhibit any such phenomena in which intrinsic material chirality is not a requisite - but for such materials, the phenomena examined in Section 3.1 will have greater significance.

It will now be highlighted how extracting the relevant terms from (7) shows a similar behaviour of discriminatory singlephoton absorption, for studies using twisted photons, in achiral molecules. The specific terms for such a process come from the E2E2 interactions.

To study the relevant terms, we first isolate those that exhibit a linear dependence on $\ell$ from (7):

$$
\Gamma_{\mathrm{E} 2 \mathrm{E} 2}^{\prime}(\ell)=\frac{I(\omega) N}{2 c \hbar^{2} \varepsilon_{o}} \frac{1}{r} f_{\ell, p}{ }^{2}(r) i \ell e_{i} \bar{e}_{k} Q_{i j}^{f 0} Q_{k l}^{f 0}\left[\frac{1}{f_{\ell, p}(r)} \frac{\partial f_{\ell, p}(r)}{\partial r}\left(\hat{\varphi}_{j} \hat{r}_{l}-\hat{\varphi}_{l} \hat{r}_{j}\right)+\frac{1}{r}\left(\hat{\varphi}_{l} \hat{r}_{j}-\hat{\varphi}_{j} \hat{r}_{l}\right)\right] .
$$

On the same physical grounds as in the E1E2 case, it is evident that the rate result must be a real quantity, and due to the quadrupole transition moments being intrinsically real, circularly polarization states of light must be invoked in order to secure a real result: 


$$
\Gamma_{\mathrm{E} 2 \mathrm{E} 2}^{\prime(\mathrm{R})}(\ell)= \pm \ell \frac{I(\omega) N}{4 c \hbar^{2} \varepsilon_{o}} \frac{1}{r} f_{\ell, p}{ }^{2}(r) \varepsilon_{i k m} \hat{k}_{m} Q_{i j}^{f 0} Q_{k l}^{f 0}\left[\frac{\partial \ln f_{\ell, p}(r)}{\partial r}\left(\hat{\varphi}_{j} \hat{r}_{l}-\hat{\varphi}_{l} \hat{r}_{j}\right)+\frac{1}{r}\left(\hat{\varphi}_{l} \hat{r}_{j}-\hat{\varphi}_{j} \hat{r}_{l}\right)\right],
$$

which may be more neatly expressed as an absolute difference of the absorption of left- and right-handed circularly polarized photons (an analogous treatment to that of (11)):

$$
\Gamma_{\mathrm{E} 2 \mathrm{E} 2}^{(\mathrm{L})}(\ell)-\Gamma_{\mathrm{E} 2 \mathrm{E} 2}^{\prime(\mathrm{R})}(\ell)=\ell \frac{I(\omega) N}{2 c \hbar^{2} \varepsilon_{o}} \frac{1}{r} f_{\ell, p}{ }^{2}(r) \varepsilon_{i k m} \hat{k}_{m} Q_{i j}^{f 0} Q_{k l}^{f 0}\left(\hat{\varphi}_{j} \hat{r}_{l}-\hat{\varphi}_{l} \hat{r}_{j}\right)\left[\frac{\partial \ln f_{\ell, p}(r)}{\partial r}-\frac{1}{r}\right] .
$$

The result secured in (15) clearly highlights its linear-dependence on $\ell$, though the remainder of the expression involves a plethora of complicated positional parameters stemming from structure of an LG beam.

\section{DISCUSSION}

This paper has highlighted how the synergy of the spin and orbital angular momentum of light leads to a discriminatory single-photon absorption rate orientated systems of both chiral and achiral molecules. This interplay between the SAM and OAM of light is an interesting manifestation of a spin-orbit interaction. ${ }^{36-38}$ In the case of chiral media, the handedness of both the light and the molecules plays a decisive role in the magnitude of the rate for the optical process (CVD). However, it has also been explicitly shown how such discriminatory effects can also occur through the handedness of the radiation alone, in systems where material chirality is not necessary (i.e. achiral molecules).

To most clearly exhibit the general form of single-photon absorption, and the potential dependence of its rate on both SAM and OAM aspects of light, the rate equation may be succinctly expressed as follows, in which the values of the coefficients $a, b$ and $c$ all depend on both beam and material properties;

$\Gamma^{(\mathrm{L} / \mathrm{R})}=a+b \sigma+c \sigma \ell$.

Here, $a$ represents all the terms which exhibit no dependence on the handedness of any part of the light-matter system typical contributions that are represented by this term include the E1E1 and M1M1. The next term $b \sigma$ is dependent on the helicity $\sigma$ of circularly polarized photons (and necessarily, in order to conserve parity, the material handedness), and as such represents the standard CD effect, dependent on both the E1M1 and E1E2 terms - the latter clearly being the specific E1E2 terms with no $\ell$ dependence. Finally, $c \sigma \ell$ represents terms due to the spin-orbit coupling, being dependent on both the helicity $\sigma$ and charge of the optical vortex $\ell$ - terms such as E1E2 (as in the CVD effect in chiral systems) and the E2E2 CVD-effect in achiral systems. The multiplicative nature of $\sigma \ell$ that is exhibited in both (10) and (14) can be seen to derive from the coupling of the spin and orbital angular momentum through $\boldsymbol{S} \cdot \boldsymbol{L}=\sigma \hbar \hat{k} \cdot \ell \hbar \hat{k}=\sigma \ell \hbar^{2}$.

To conclude, this work has highlighted how electric quadrupole transitions are a prerequisite to observing chiroptical interactions that exhibit a dependence on the vortex charge $\ell$. The rate equations have been derived for two mechanisms 
highlighting how, for anisotropic materials composed of chiral or achiral molecules, the effect can be identified as a manifestation of the spin-orbit interaction of light.

Future work now aims to probe deeper into the physicality of this spin-orbit interaction, along with extending the theory to account for a chiroptical response using twisted light beams with linear polarization - the examination of such a scheme is warranted to secure an explanation for other recent experimental observations. ${ }^{20}$ Furthermore, an extension of the theory to account for vortex charge-sensitive interactions in more elaborate optical processes also warrants further exploration. Indeed, recent experiments suggest a role in circular differential Raman scattering. ${ }^{39}$ In this whole sphere of optics, it is encouraging to see theory and experiment progressing with unusual but very welcome synchronicity.

\section{REFERENCES}

[1] Andrews, D. L. and Babiker, M., [The Angular Momentum of Light], Cambridge University Press, Cambridge, UK (2013).

[2] Beth, R. A., "Mechanical detection and measurement of the angular momentum of light," Phys. Rev. 50, 115125 (1936).

[3] Kliger, D. S. and Lewis, J. W., [Polarized Light in Optics and Spectroscopy], Elsevier Science, (2012).

[4] Allen, L., Beijersbergen, M. W., Spreeuw, R. J. C. and Woerdman, J. P., "Orbital angular momentum of light and the transformation of Laguerre-Gaussian laser modes," Phys. Rev. A 45, 8185-8189 (1992).

[5] Barnett, S. M., Babiker, M. and Padgett, M. J., [Optical orbital angular momentum] The Royal Society, (2017).

[6] Rubinsztein-Dunlop, H., Forbes, A., Berry, M., Dennis, M., Andrews, D. L., Mansuripur, M., Denz, C., Alpmann, C., Banzer, P. and Bauer, T., "Roadmap on structured light," J. Opt. 19, 013001 (2016).

[7] Bradshaw, D. S., Forbes, K. A., Leeder, J. M. and Andrews, D. L., "Chirality in optical trapping and optical binding," Photonics 2, 483-497 (2015).

[8] Forbes, K. A. and Andrews, D. L., "Chiral discrimination in optical binding," Phys. Rev. A 91, 053824 (2015).

[9] Craig, D. and Thirunamachandran, T., "New approaches to chiral discrimination in coupling between molecules," Theor. Chem. Acc. 102, 112-120 (1999).

[10] Salam, A., [Molecular Quantum Electrodynamics. Long-Range Intermolecular Interactions], Wiley, Hoboken, NJ (2010).

[11] Salam, A. and Meath, W. J., "On enantiomeric excesses obtained from racemic mixtures by using circularly polarized pulsed lasers of varying durations," Chem. Phys. 228, 115-129 (1998).

[12] Andrews, D. L., Dávila Romero, L. C. and Babiker, M., "On optical vortex interactions with chiral matter," Opt. Commun. 237, 133-139 (2004).

[13] Andrews, D. L. and Babiker, M., "Quantum electrodynamics, angular momentum and chirality," in [The Angular Momentum of Light], D. L. Andrews and M. Babiker, eds. (Cambridge University Press, New York, 2012), pp. 246-263.

[14] Araoka, F., Verbiest, T., Clays, K. and Persoons, A., "Interactions of twisted light with chiral molecules: an experimental investigation," Phys. Rev. A 71, 055401 (2005).

[15] Löffler, W., Broer, D. and Woerdman, J., "Circular dichroism of cholesteric polymers and the orbital angular momentum of light," Phys. Rev. A 83, 065801 (2011).

[16] Zambrana-Puyalto, X., Vidal, X. and Molina-Terriza, G., "Angular momentum-induced circular dichroism in non-chiral nanostructures," Nat. Commun. 5, 4922 (2014).

[17] Afanasev, A., Carlson, C. E. and Solyanik, M., "Circular Dichroism of Twisted Photons in the Non-Chiral Atomic Matter,” J. Opt. 19, 105401 (2017).

[18] Valev, V. K., Baumberg, J. J., Sibilia, C. and Verbiest, T., "Chirality and chiroptical effects in plasmonic nanostructures: fundamentals, recent progress, and outlook," Adv. Mater. 25, 2517-2534 (2013).

[19] Roder, P. B., Manandhar, S., Smith, B. E., Zhou, X., Shutthanandan, V. S. and Pauzauskie, P. J., "Photothermal superheating of water with ion-implanted silicon nanowires," Advanced Optical Materials 3, 1362-1367 (2015).

[20] Brullot, W., Vanbel, M. K., Swusten, T. and Verbiest, T., "Resolving enantiomers using the optical angular momentum of twisted light," Science Advances 2, e1501349 (2016). 
[21] Forbes, K. A. and Andrews, D. L., “Optical orbital angular momentum: twisted light and chirality,” Opt. Lett. 43, 435-438 (2018).

[22] Forbes, K. A. and Andrews, D. L., "Chiroptical interactions between twisted light and chiral media," Proc. SPIE 10549, 1054915 (2018).

[23] Andrews, D. L., Jones, G. A., Salam, A. and Woolley, R. G., "Perspective: Quantum Hamiltonians for optical interactions," J. Chem. Phys. 148, 040901 (2018).

[24] Craig, D. P. and Thirunamachandran, T., [Molecular Quantum Electrodynamics: An Introduction to RadiationMolecule Interactions], Dover Publications, Mineola, NY (1998).

[25] Forbes, K. A., Bradshaw, D. S. and Andrews, D. L., "Identifying diamagnetic interactions in scattering and nonlinear optics," Phys. Rev. A 94, 033837 (2016).

[26] Dávila Romero, L. C., Andrews, D. L. and Babiker, M., “A quantum electrodynamics framework for the nonlinear optics of twisted beams," J. Opt. B: Quantum Semiclass. Opt. 4, S66-S72 (2002).

[27] Forbes, K. A., [A Quantum Electrodynamical Approach To Chirality And Photonics: Nonlinear Optics, Structured Light, And Optical Forces] University of East Anglia, Norfolk, U.K.(2018).

[28] Power, E. and Thirunamachandran, T., "Circular dichroism: A general theory based on quantum electrodynamics,” J. Chem. Phys. 60, 3695-3701 (1974).

[29] Andrews, D. L., "Quantum formulation for nanoscale optical and material chirality: symmetry issues, space and time parity, and observables,” J. Opt. 20, 033003 (2018).

[30] Lembessis, V. E. and Babiker, M., "Enhanced quadrupole effects for atoms in optical vortices," Phys. Rev. Lett. 110,083002 (2013).

[31] Afanasev, A., Carlson, C. E. and Mukherjee, A., "High-multipole excitations of hydrogen-like atoms by twisted photons near a phase singularity," J. Opt 18, 074013 (2016).

[32] Wang, Z., Cheng, F., Winsor, T. and Liu, Y., "Optical chiral metamaterials: a review of the fundamentals, fabrication methods and applications," Nanotechnology 27, 412001 (2016).

[33] Mason, W. R., [Magnetic Circular Dichroism Spectroscopy], John Wiley \& Sons, (2007).

[34] Craig, D., Power, E. and Thirunamachandran, T., "The dynamic terms in induced circular dichroism," Proc. R. Soc. A 348, 19-38 (1976).

[35] Claborn, K., Isborn, C., Kaminsky, W. and Kahr, B., "Optical rotation of achiral compounds,” Angew. Chem. Int. Ed. 47, 5706-5717 (2008).

[36] Cardano, F. and Marrucci, L., "Spin-orbit photonics," Nat. Photonics 9, 776 (2015).

[37] Bliokh, K. Y., Rodríguez-Fortuño, F., Nori, F. and Zayats, A. V., "Spin-orbit interactions of light,” Nat. Photonics 9, 796-808 (2015).

[38] Samlan, C., Suna, R. R., Naik, D. N. and Viswanathan, N. K., "Spin-orbit beams for optical chirality measurement," Appl. Phys. Lett. 112, 031101 (2018).

[39] Bendau, E., Zhang, L., Gozali, R., Ashrafi, S. and Alfano, R. R., "Vortex beams and optical activity of sucrose." Proc. SPIE 10120, 5. 